\title{
Crosslinked hyaluronic acid with liposomes and crocin for management symptoms of dry eye disease caused by moderate meibomian gland dysfunction
}

\author{
José-María Sánchez-González, Concepción De-Hita-Cantalejo, María Carmen Sánchez- \\ González
}

Department of Physics of Condensed Matter, Optics Area, University of Seville, Seville 41012, Spain

Correspondence to: José-María Sánchez-González. Reina Mercedes St. Physic Faculty, University of Seville, Seville 41012, Spain. Jsanchez80@us.es

Received: 2020-02-13 Accepted: 2020-05-28

\begin{abstract}
- AIM: To study the effect of uncrosslinked and crosslinked hyaluronic acid combined with other artificial tear components in patients with dry eye caused by moderate meibomian gland dysfunction.
\end{abstract}

- METHODS: Prospective, single-blind, contralateral eye study. Fifty eyes (25 patients) were analyzed. Eye selection for each tear type was random, and the eye drop formulations, $0.4 \%$ uncrosslinked hyaluronic acid and $0.2 \%$ galactoxyloglucan (tear A) and $0.15 \%$ crosslinked hyaluronic acid, crocin, and liposomes (tear B) were used. The determined dosing schedule was three times a day for six weeks, and the study participants underwent a clinical examination before and $45 \mathrm{~d}$ after lubricant treatment. The Schirmer test, tear breakup time (TBUT) test, and Ocular Surface Disease Index (OSDI) questionnaire were applied before and after instillation period with both types of artificial tears.

- RESULTS: On the Schirmer test, a significant improvement was obtained with both tear $\mathrm{A}(P<0.01)$ and tear $\mathrm{B}(P<0.01)$. On the TBUT test, a significant improvement was obtained with tear $\mathrm{A}(P<0.01)$ and tear $\mathrm{B}(P<0.01)$. The OSDI score significantly decreased after instillation period with both artificial tear types $(P<0.01)$.

- CONCLUSION: Uncrosslinked hyaluronic acid combined with other components, such as tamarind seed polysaccharide, and crosslinked hyaluronic acid combined with liposomes and crocin are effective for management symptoms of dry eye disease.

- KEYWORDS: liposome; crosslinked hyaluronic acid; meibomian gland dysfunction; contact lens; dry eye disease DOI:10.18240/ijo.2020.09.05
Citation: Sánchez-González JM, De-Hita-Cantalejo C, SánchezGonzález MC. Crosslinked hyaluronic acid with liposomes and crocin for management symptoms of dry eye disease caused by moderate meibomian gland dysfunction. Int J Ophthalmol 2020;13(9):1368-1373

\section{INTRODUCTION}

Dy eye has recently been defined as a multifactorial disease $^{[1-3]}$. Loss of homeostasis of the tear film is a pathophysiological factor by which dry eye is classified according to its etiology as either aqueous-deficient or evaporative dry eye, with each being a continuation of the other ${ }^{[2-4]}$. Evaporative dry eye results from excessive loss of the aqueous layer, attributed to a deficient or unstable lipid layer, which controls evaporation of the tear film. The most common cause of evaporative dry eye is meibomian gland dysfunction $(\mathrm{MGD})^{[5-6]}$, which occurs in over $85 \%$ of dry eye disease. Blepharitis, low blink rate, drug action Accutane, vitamin A deficiency or contact lens wear were other causes of MGD ${ }^{[2,4]}$. Abnormal lipid composition affects the physicochemical properties of the tear film and therefore its stability. The secretion meibomian was secreted by normal meibomian gland $^{[7]}$, composed of lipids mostly. The secretion meibomian biochemical compositions are extremely complex and more than ninety different proteins were identified in meibomian gland secretions ${ }^{[8]}$. In MGD, the secretion meibomian is deficient in quality or quantity.

Currently, the most common method to temporarily manage symptoms and temporarily supplement the tear layer is to use over-the-counter drops. These lubricants assume a deficiency in the lipid component of the tear film. In patients with evaporative dry eye, they cause a temporary renewal of the tear film but in some cases can increase tear evaporation. In these cases, lubricants are applied more frequently, involving discomfort and added cost for the patient.

In recent years, interest in using lipid-containing lubricants has increased, and thus, these products have been extensively commercialized. New formulations include $0.6 \%$ propylene glycol (Systane Balance, Alcon, Fort Worth, Texas, USA), $0.18 \%$ sodium hyaluronate with triglycerides, phospholipids 
and lipoic acid (Neovis Total Multi, Horus Pharma, SaintLaurent du Var, France), and sodium carmellose with glycerin (Optive Advanced, Allergan, Irvine, California, USA).

Since the discovery that hyaluronic acid can act as an ocular lubricant, it has been included in many artificial tear formulations. The viscoelasticity of this polysaccharide increases tear film stability, consequently reducing dry eye symptoms ${ }^{[9-11]}$. The high viscosity of the polysaccharide is largely due to the molecular weight of the hyaluronic acid chain. Crosslinked hyaluronic acid confers a higher molecular weight to the polysaccharide chain and has been investigated in recent studies, used alone and combined with other elements ${ }^{[12-13]}$. Previous authors ${ }^{[14-17]}$ study the efficacy of hyaluronic acid alone and this supposes a low permanence of the tear on the ocular surface, so that the combination of hyaluronic acid with other components compatible with tear can lead to an increase in moisturizing properties and also a significant reduction in tear evaporation time. Furthermore, lubricants without a lipid component only replace the aqueous layer of the tear without providing a first lipid protective layer ${ }^{[18]}$, so that the evaporation of said aqueous layer is greater without a regulatory lipid barrier ${ }^{[19]}$.

Galactoxyloglucan is a natural polysaccharide derived from the tamarind seed mucin layer. The molecular structure of galactoxyloglucan confers its mucoadhesive properties, allowing xyloglucan formulations to act as a barrier that reduces bacterial adhesion and invasion and preserves tight junctions and paracellular flow, as observed in several in vitro and in vivo studies ${ }^{[20]}$. Thus, xyloglucan possesses protective properties enabling it to form a barrier film and is a nonpharmacological alternative for treatment of various diseases, such as dry eye. Tamarind seed polysaccharide has been used for the symptom's management of dry eye, and its efficacy and ocular surface tolerability have been verified. Rolando and Valente ${ }^{[21]}$ showed that $0.5 \%$ tamarind seed polysaccharide had an effect equivalent to that produced by $0.2 \%$ hyaluronic acid. In our case, the artificial tear formulation included both components, and thus, an added effect was observed in dry eye improvement. From a chemical point of view, excellent synergistic polysaccharide interactions have been found between tamarind seed polysaccharide and hyaluronic acid ${ }^{[18]}$. Tamarind seed polysaccharide can noncovalently stabilize hyaluronic acid; in this same study, Uccello-Barretta et al ${ }^{[18]}$ determined a minimum concentration of $1.5 \mathrm{mg} / \mathrm{mL}$ of each polysaccharide to generate a stable union. Lipid-based eye drops are a new alternative to aqueous-based artificial tears because they closely to tear film composition. Lipid not only relieve dry eye disease symptoms immediately after topical administration, but improve the lipid tear film structure and thickness, resulting in enhanced tear film stability ${ }^{[19]}$. Crocin, natural carotenoid compounds, which have been reported to possess anti-inflammatory, antioxidative, and neuroprotective effects in ocular diseases (e.g., in age related macular degeneration, glaucoma, and diabetic maculopathy) $)^{[22]}$ has been included to hyaluronic acid to find out if these properties are extensible in an artificial tear.

The purpose of this study was to evaluate the clinical efficacy of $0.4 \%$ uncrosslinked hyaluronic acid and $0.2 \%$ galactoxyloglucan (tear A) and $0.15 \%$ crosslinked hyaluronic acid, crocin, and liposomes (tear B) in dry eye disease caused by meibomian gland dysfunction. To the best of our knowledge, this is the first study to report the clinical effect of crosslinked hyaluronic acid, liposomes and crocin combination.

\section{SUBJECTS AND METHODS}

Ethical Approval This is a prospective, single-blind, contralateral eye study, conducted between December $1^{\text {st }}$, 2017, and March $31^{\text {st }}, 2018$. The study was carried out in the facilities of the School of Pharmacy (Optics and Optometry Departments) at the University of Seville. The principles of the Declaration of Helsinki were followed. the study has been registered as a clinical trial at www.clinicaltrial.gov (No. NCT03617315). All patients read, understood, and signed an informed consent form prior to participation in the study.

Subjects Fifty eyes were analyzed (25 patients). The subjects selected were over 18 years of age. No gender distinction was made in the selection of subjects. All subjects were silicone hydrogel contact lens wearers on a monthly replacement schedule. Patients included in both arms of the study reported using electronic devices between 6 and $8 \mathrm{~h}$ a day. The subjects had no history of eye disease or previous eye surgery and were not taking systemic or ocular medications.

Materials A slit lamp (TOPCON SL-6E, Japan) was used to study the tear film, and fluorescein strips (Bio Glo ContaCare Ophthalmics \& Diagnostics, Gujarat, India) impregnated with saline solution were used for the tear breakup time (TBUT) test. Next, tears were examined with the cobalt blue filter of the slit lamp. TBUT strips and dry sterile applicators have been used. Subjects blinked naturally three times and then to stop blinking until instructed. Measurement was the break of time that elapses between a complete blink and the presence of the initial disruption in the tear film. The Schirmer test (Tear Flo, HUB Pharmaceutical, Michigan, USA) was used to measure tear volume. The Schirmer strip was placed between the outer and middle third of the lower eyelid. After $5 \mathrm{~min}$, the wetted length was measured in millimeters. The location, time, and humidity conditions were the same for all patients examined.

The patients also completed the Ocular Surface Disease Index (OSDI) questionnaire during the first visit along with a questionnaire inquiring about their personal data, type 


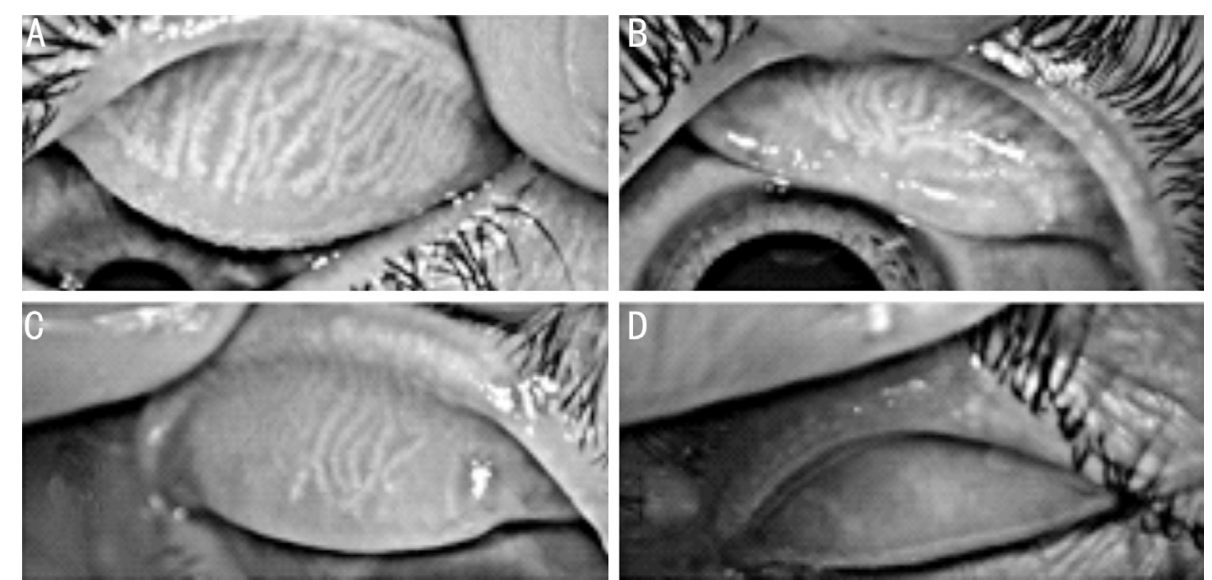

Figure 1 MGD patterns according to the Meiboscore, which was based on the percentage of meibomian gland loss determined using image processing software A: Grade 1 MGD of the upper eyelid; B: Grade 2 MGD of the upper eyelid; C: Grade 3 MGD of the upper eyelid; D: Grade 4 MGD of the upper eyelid.

of contact lenses used, and hours of wear time per day. To evaluate the meibomian glands, infrared meibography was performed with a COBRA ${ }^{\circledR}$ HD fundus camera (Construzione Strumenti Oftalmici, Firenze, Italy). MGD degree was measured by Image $\mathrm{J}$ method defined by Pult and Nichols ${ }^{[23]}$. MGD was classified as one of four grades according to the severity of the loss (Figure 1). MGD score of superior lids strong correlates with percent of superior lids meibomian loss $(r=0.85, P<0.01)$ and MGD score of inferior lids strong correlates with percent of inferior lid meibomian loss $(r=0.92$, $P<0.01)$.

Before the start of the study, the TBUT, Schirmer score, OSDI score, percentage of gland loss, and MGD grade were measured. After follow-up period, the TBUT, Schirmer score, and OSDI score were measured again. Meibomian gland loss was not re-measured because ocular lubricants do not alter the glandular pattern. A change in grade of Meiboscore was not expected and thus not gathered. In this sense, the function of the meibomian glands was not evaluated either. There was not any change in environmental conditions at the follow up appointment. Patients did not know the type of artificial tear instilled in each eye during the measurements.

Procedure The contralateral eye was used to establish the two arms of the study to evaluate the two artificial tears. Eye selection for each artificial tear formulation was random, using a random number table, and the formulations were named artificial tear A and artificial tear B. Patients were not told the type of artificial tears they would use or their difference, and therefore, they were unaware of the possible benefits of such tears. The examiner knew the tears applied to each eye. The absence of ocular disease was analyzed by a complete examination of the ocular surface, intraocular pressure, and fundus of the eye.

Regarding the lubricants, artificial tear A (Aquoral Forte ${ }^{\circledR}$, ESTEVE $^{\circledR}$, Farmigea, Pisa, Italy) is a mixture of $0.4 \%$ uncrosslinked hyaluronic acid and $0.2 \%$ galactoxyloglucan. The galactoxyloglucan is extracted from the tamarind seed. The package contains 30 single $0.5-\mathrm{mL}$ doses. The doses are packaged for daily use, and thus, the lubricant cannot be used if $12 \mathrm{~h}$ have passed since the dose dispenser was opened. It is a preservative-free lubricant.

Tear B [Aquoral Lipo ${ }^{\circledR}$ (Spain)/Lumixa ${ }^{\circledR}$ (Italy), ESTEVE ${ }^{\circledR}$, Farmigea, Pisa, Italy] is a mixture of three components: $0.15 \%$ crosslinked hyaluronic acid, crocin, and liposomes. It is an ophthalmic lubricant and antioxidant solution. The package is a $10-\mathrm{mL}$ multidose container, and thus, the lubricant can be used for a prolonged period. The lubricant is composed of liposomes, $0.15 \%$ crosslinked hyaluronic acid sodium salt, ethylenediaminetetraacetic acid (EDTA) disodium salt, and crocin. Although this tear comes in a multidose container which impedes microorganisms from penetrating the solution inside. Contact lenses can be used while both lubricants are applied, and the study patients wore their monthly silicone hydrogel contact lenses throughout the study period.

All patients in the study spent one month using no artificial tears or eye drops. Once this 'wash out' period ended, patients were given instructions on the proper instillation technique for artificial tears. The artificial tears were administered three times a day for six weeks, and the study subjects underwent a clinical examination before and $45 \mathrm{~d}$ after lubricant period. Patients were instructed not to use any other lubricant or ocular therapy during the study period. Patient compliance was tracked with a weekly email reminder. The initial tests were repeated; however, meibography was not repeated because artificial tears were not expected to cause growth or modification of the meibomian glands.

Statistical Analysis The data were analyzed using the SPSS 24 package for Windows (SPSS Science, Chicago, IL, USA). The normality of the variables was verified using the ShapiroWilk test. A descriptive data analysis technique was developed, 

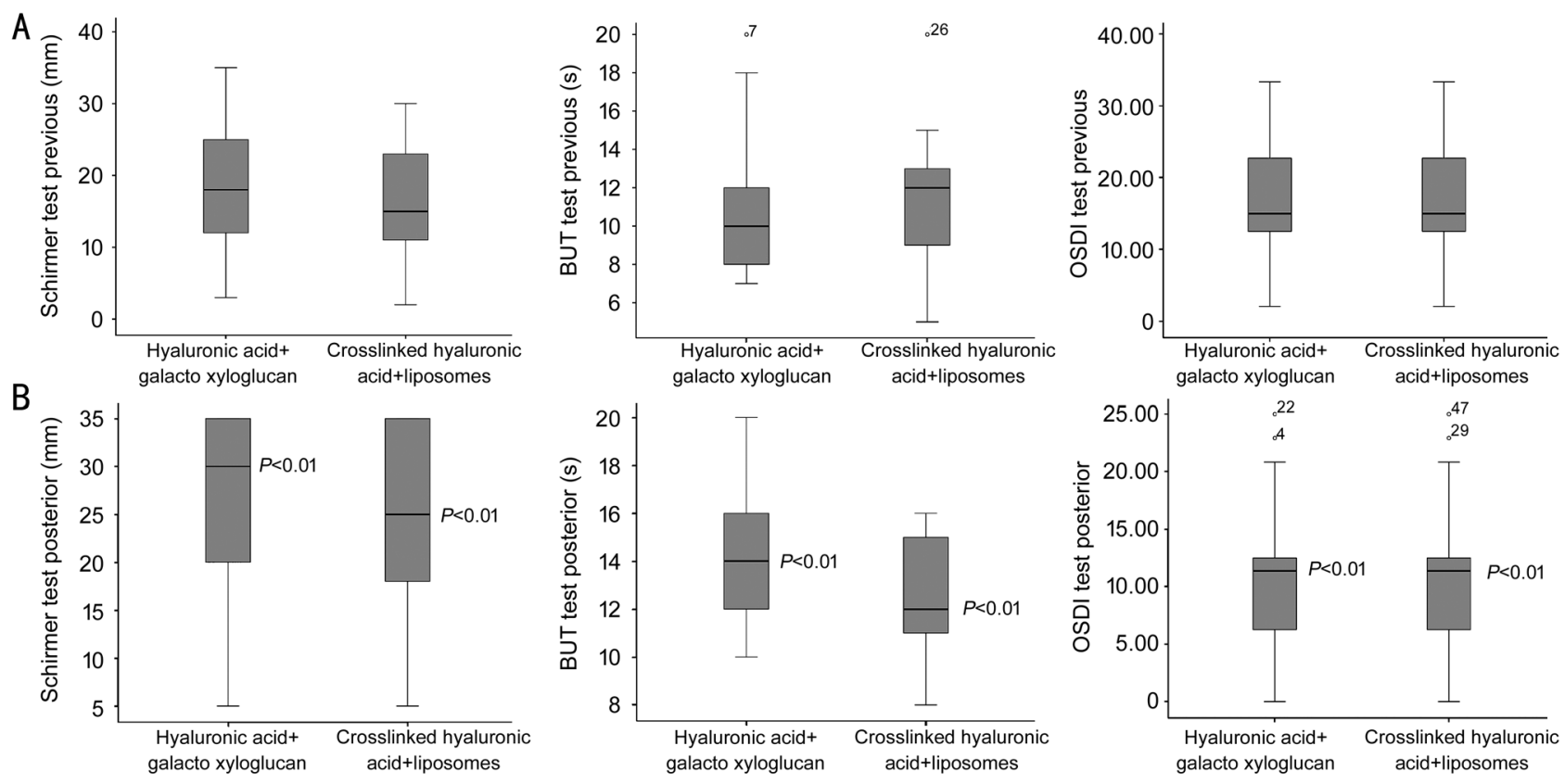

Figure 2 Comparative box and whisker plots between the tear A and tear B A: the Schirmer test, TBUT, and OSDI score before treatment; B: The Schirmer test, TBUT, and OSDI score after treatment. Wilcoxon test was used for related nonparametric variables.

Table 1 Descriptive characteristics of the patients in the study before treatment with artificial tears

\begin{tabular}{lccc}
\hline Characteristics & Tear A group $(n=25)$ & Tear B group $(n=25)$ & $P$ \\
\hline Sex (M/F) & $5 / 20$ & $5 / 20$ & 1.00 \\
Age (y) & $21.76 \pm 1.39(19.0-25.0)$ & $21.76 \pm 1.39(19.0-25.0)$ & 1.00 \\
Intraocular pressure (mm Hg) & $13.1 \pm 1.8(11.0-15.0)$ & $13.9 \pm 2.0(10.0-16.0)$ & $0.32^{\mathrm{b}}$ \\
Upper meibomian gland loss (\%) & $24.60 \pm 9.55(8.7-42.8)$ & $29.38 \pm 10.77(7.0-47.7)$ & $0.12^{\mathrm{a}}$ \\
MGD grade (Meiboscore), upper eyelid & $1.44 \pm 0.58(0-2)$ & $1.60 \pm 0.57(0-2)$ & $0.28^{\mathrm{a}}$ \\
Lower meibomian gland loss (\%) & $49.88 \pm 15.90(20.8-84.7)$ & $46.04 \pm 15.78(22.0-84.8)$ & $0.29^{\mathrm{a}}$ \\
MGD grade (Meiboscore), lower eyelid & $2.48 \pm 0.71(1-4)$ & $2.40 \pm 0.64(1-4)$ & $0.56^{\mathrm{a}}$ \\
Schirmer test value (mm) & $18.08 \pm 8.99(3-35)$ & $16.76 \pm 7.86(2-30)$ & $0.55^{\mathrm{a}}$ \\
TBUT (s) & $10.60 \pm 3.40(7-20)$ & $11.24 \pm 3.35(5-20)$ & $0.27^{\mathrm{a}}$ \\
OSDI score & $16.67 \pm 8.47(2.08-33.33)$ & $16.67 \pm 8.47(2.08-33.33)$ & 1.00 \\
\hline
\end{tabular}

A significance of 1.00 shows no difference between one group and another because the contralateral eye was used. ${ }^{a}$ Mann-Whitney $U$ test;

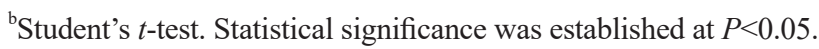

showing the count and proportion in each category of the qualitative variables and the mean and standard deviation (SD); failing this, the median and interquartile range of the quantitative variables is given. All statistical tests were performed with a $95 \%$ confidence level $(P<0.05)$.

\section{RESULTS}

Fifty eyes were examined. Since this was a contralateral study, one of the eyes was treated with tear $\mathrm{A}(n=25)$ and the other with tear B $(n=25)$. Table 1 shows the characteristics of the two study groups before treatment with artificial tears. Since the study is a contralateral design study treatment and control group did not achieve statistically differences for any of the variables. Sex, age, and OSDI score, the statistical significance is 1.00 when it is the same patient. In addition, intraocular pressure, MGD, Schirmer and TBUT showed not significant differences between both groups.
For the Schirmer test variable, a significant improvement was obtained for both tear A $(P<0.01)$ and tear $\mathrm{B}(P<0.01)$, which corresponds to an improvement in aqueous tear volume. Likewise, for TBUT, a significant improvement was obtained with the tear composed of hyaluronic acid and galactoxyloglucan $(P<0.01)$ and the tear composed of crosslinked hyaluronic acid and liposomes $(P<0.01)$. This variable evaluates the stability of the lipid component of the tear film. Lastly, the OSDI score decreased significantly $(P<0.01)$ after treatment with both artificial tears. Here, the results could not be measured monocularly because many respondents did not know how to differentiate between the two eyes for many of the questions asked in the OSDI questionnaire. The results are summarized in Figure 2.

Both treatments were found to be safe and well tolerated by the patients during the three instillations per day and throughout 
the $45 \mathrm{~d}$. No changes were found in intraocular pressure. All patients maintained their maximum corrected vision after the study. In a survey of the patients after using both ophthalmic lubricants, $12 \%$ of the respondents reported they had blurred vision with tear B. These symptoms of blurring disappeared quickly according to the recorded responses. Another issue addressed was the itching sensation. While only $8 \%$ of patients reported itching with tear $\mathrm{A}$, this result increased to $32 \%$ of respondents with tear B. Finally, there were no adverse events.

\section{DISCUSSION}

This study evaluated the efficiency of two hyaluronic acidbased artificial tear dry eye disease symptom's management of moderate MGD. Our results showed significant improvement in the three variables analyzed: Schirmer test values $(P<0.01)$, TBUT $(P<0.01)$, and OSDI score $(P<0.01)$ for both tears A and $\mathrm{B}$. The upper eyelid exhibited less loss in both the right eye and left eye $[24.60 \pm 9.55(8.7-42.8)$ and $29.38 \pm 10.77$ (7.0-47.7), respectively]. In the lower eyelid, gland loss was greater [49.88 $\pm 15.90(20.8-84.7)$ and $46.04 \pm 15.78$ (22.0-84.8), right and left eye, respectively]. Arita et $a l^{[24]}$ used infrared meibography to observe gland morphology with the eyelid everted. The results obtained support our findings. They also described and classified gland loss using a 5-grade scale called the meiboscore ${ }^{[25]}$. This scale was based on the percentage of meibomian gland loss.

This is the first time the latter artificial tear formulation has been the subject of a clinical study. Our results showed a significant improvement in the Schirmer test values, TBUT, and OSDI scores for both tear types. Previous studies have described the improvement achieved by hyaluronic acid combined with galactoxyloglucan. Barabino et $a l^{[26]}$ found that the galactoxyloglucan-based artificial tear was a good tear substitute. In addition, they concluded that the effect of galactoxyloglucan with hyaluronic acid on the tear is greater than that of hyaluronic acid alone and greater than carmellose. Their results, which are in line with ours, suggest that noncovalent interaction of galactoxyloglucan with other components improves treatment outcomes compared with use of the polymer alone.

Use of the other artificial tear, containing crosslinked hyaluronic acid combined with liposomes and crocin, led to a significant improvement in the Schirmer test values, TBUT, and OSDI scores, along with an improvement in the initial symptoms. A wide variety of tears contain a lipid component, for example, homogenized oil eye drops, $1.25 \%$ castor oil emulsion, and carbomer-based lipid-containing gel. All these lubricants have been shown to improve the symptoms and signs of MGD under certain conditions. A summary of the studies conducted with these lubricants is included in a review by Lee and Tong ${ }^{[27]}$. A more recent review by Garrigue et $a l^{[19]}$ examines the currently marketed artificial tears. This review addresses aspects such as the addition of liposomes to ocular lubricants as a natural substitute for the lipid layer of the tear film. Itching was more frequent in Tear B because of the preservative (EDTA) present in it.

Garrigue $e t a l^{[19]}$ also stated that lipid-based therapies are an attractive alternative to water-based artificial tears because they more closely resemble the tear film composition. Lipid-based therapies not only eliminate patient symptoms immediately after topical administration but also improve the lipid structure of the tear film, resulting in improved tear film stability. Another study also tested new lipid-based formulations for the effective symptoms management of dry eye ${ }^{[28]}$ and for replacing the lipid layer of the tear film. More recently, lipidcontaining gels have been described, producing results in accordance with ours ${ }^{[29]}$.

Studies examining the effect of crosslinked hyaluronic acid on dry eye are scarce. Moreover, these studies report combinations with components different from those of tear B in our study. In the first study conducted in vitro ${ }^{[12]}$, corneal epithelialization improved with two artificial tear formulations containing crosslinked hyaluronic acid combined with $0.02 \%$ and $0.4 \%$ urea; both compounds quantitatively improved the ocular surface. A recently published study examined the efficacy of an artificial tear containing crosslinked hyaluronic acid combined with coenzyme Q10 ${ }^{[13]}$ and concluded that the resulting effect on the ocular surface is greater than that with hyaluronic acid alone. The hyaluronic acid/coenzyme Q10 combination led to a reduction in the OSDI score and in corneal and conjunctival staining.

In conclusion, uncrosslinked hyaluronic acid combined with other components, such as tamarind seed polysaccharide, and crosslinked hyaluronic acid combined with liposomes and crocin are effective for symptoms management of dry eye.

\section{ACKNOWLEDGEMENTS}

Conflicts of Interest: Sánchez-González JM, None; DeHita-Cantalejo C, None; Sánchez-González MC, None.

\section{REFERENCES}

1 Nelson JD, Craig JP, Akpek EK, Azar DT, Belmonte C, Bron AJ, Clayton JA, Dogru M, Dua HS, Foulks GN, Gomes JAP, Hammitt KM, Holopainen J, Jones L, Joo CK, Liu ZG, Nichols JJ, Nichols KK, Novack GD, Sangwan V, Stapleton F, Tomlinson A, Tsubota K, Willcox MDP, Wolffsohn JS, Sullivan DA. TFOS DEWS II introduction. Ocul Surf 2017;15(3):269-275.

2 Craig JP, Nichols KK, Akpek EK, Caffery B, Dua HS, Joo CK, Liu ZG, Nelson JD, Nichols JJ, Tsubota K, Stapleton F. TFOS DEWS II definition and classification report. Ocul Surf 2017;15(3):276-283.

3 Stapleton F, Alves M, Bunya VY, Jalbert I, Lekhanont K, Malet F, Na KS, Schaumberg D, Uchino M, Vehof J, Viso E, Vitale S, Jones L. TFOS DEWS II epidemiology report. Ocul Surf 2017;15(3):334-365. 
Int J Ophthalmol， Vol. 13， No. 9，Sep.18，2020 www.ijo.cn

Tel: 8629-82245172 8629-82210956 Email: ijopress@163.com

4 Wolffsohn JS, Arita R, Chalmers R, Djalilian A, Dogru M, Dumbleton K, Gupta PK, Karpecki P, Lazreg S, Pult H, Sullivan BD, Tomlinson A, Tong L, Villani E, Yoon KC, Jones L, Craig JP. TFOS DEWS II Diagnostic Methodology Report. Ocul Surf 2017;15(3):539-574.

5 García-Resúa C, Pena-Verdeal H, Giráldez MJ, Yebra-Pimentel E. Clinical relationship of meibometry with ocular symptoms and tear film stability. Cont Lens Anterior Eye 2017;40(6):408-416.

6 Turnbull PRK, Misra SL, Craig JP. Comparison of treatment effect across varying severities of meibomian gland dropout. Cont Lens Anterior Eye 2018;41(1):88-92.

7 Nicolaides N, Kaitaranta JK, Rawdah TN, Macy JI, Boswell FM 3rd, Smith RE. Meibomian gland studies: comparison of steer and human lipids. Invest Ophthalmol Vis Sci 1981;20(4):522-536.

8 Tsai PS, Evans JE, Green KM, Sullivan RM, Schaumberg DA, Richards SM, Dana MR, Sullivan DA. Proteomic analysis of human meibomian gland secretions. Br J Ophthalmol 2006;90(3):372-377.

9 Mateo Orobia AJ, Saa J, Ollero Lorenzo A, Herreras JM. Combination of hyaluronic acid, carmellose, and osmoprotectants for the treatment of dry eye disease. Clin Ophthalmol 2018;12:453-461.

10 Doan S, Bremond-Gignac D, Chiambaretta F. Comparison of the effect of a hyaluronate-trehalose solution to hyaluronate alone on Ocular Surface Disease Index in patients with moderate to severe dry eye disease. Curr Med Res Opin 2018;34(8):1373-1376.

11 Kim YH, Kang YS, Lee HS, Choi W, You IC, Yoon KC. Effectiveness of combined tear film therapy in patients with evaporative dry eye with short tear film breakup time. J Ocul Pharmacol Ther 2017;33(8):635-643.

12 Fallacara A, Vertuani S, Panozzo G, Pecorelli A, Valacchi G, Manfredini S. Novel artificial tears containing cross-linked hyaluronic acid: an in vitro re-epithelialization study. Molecules 2017;22(12):E2104.

13 Postorino EI, Rania L, Aragona E, Mannucci C, Alibrandi A, Calapai G, Puzzolo D, Aragona P. Efficacy of eyedrops containing cross-linked hyaluronic acid and coenzyme Q10 in treating patients with mild to moderate dry eye. Eur J Ophthalmol 2018;28(1):25-31.

14 Pinto-Fraga J, López-de la Rosa A, Blázquez Arauzo F, Urbano Rodríguez R, González-García MJ. Efficacy and safety of $0.2 \%$ hyaluronic acid in the management of dry eye disease. Eye Contact Lens 2017;43(1):57-63.

15 López-de la Rosa A, Pinto-Fraga J, Blázquez Arauzo F, Urbano Rodríguez R, González-García MJ. Safety and efficacy of an artificial tear containing $0.3 \%$ hyaluronic acid in the management of moderateto-severe dry eye disease. Eye Contact Lens 2017;43(6):383-388.

16 Groß D, Childs M, Piaton JM. Comparison of $0.2 \%$ and $0.18 \%$ hyaluronate eye drops in patients with moderate to severe dry eye with keratitis or keratoconjunctivitis. Clin Ophthalmol 2017;11:631-638.

17 Mencucci R, Boccalini C, Caputo R, Favuzza E. Effect of a hyaluronic acid and carboxymethylcellulose ophthalmic solution on ocular comfort and tear-film instability after cataract surgery. J Cataract Refract Surg 2015;41(8):1699-1704.

18 Uccello-Barretta G, Balzano F, Vanni L, Sansò M. Mucoadhesive properties of tamarind-seed polysaccharide/hyaluronic acid mixtures: a nuclear magnetic resonance spectroscopy investigation. Carbohydr Polym 2013;91(2):568-572.

19 Garrigue JS, Amrane M, Faure MO, Holopainen JM, Tong L. Relevance of lipid-based products in the management of dry eye disease. J Ocul Pharmacol Ther 2017;33(9):647-661.

20 Piqué N, Gómez-Guillén MDC, Montero MP. Xyloglucan, a plant polymer with barrier protective properties over the mucous membranes: an overview. Int J Mol Sci 2018;19(3):673.

21 Rolando M, Valente C. Establishing the tolerability and performance of tamarind seed polysaccharide (TSP) in treating dry eye syndrome: results of a clinical study. BMC Ophthalmol 2007;7:5.

22 Heitmar R, Brown J, Kyrou I. Saffron (Crocus sativus L.) in ocular diseases: a narrative review of the existing evidence from clinical studies. Nutrients 2019;11(3):649.

23 Pult H, Nichols JJ. A review of meibography. Optom Vis Sci 2012;89(5):E760-E769.

24 Arita R, Fukuoka S, Morishige N. New insights into the morphology and function of meibomian glands. Exp Eye Res 2017;163:64-71.

25 Arita R, Morishige N, Koh S, Shirakawa R, Kawashima M, Sakimoto T, Suzuki T, Tsubota K. Increased tear fluid production as a compensatory response to meibomian gland loss: a multicenter crosssectional study. Ophthalmology 2015;122(5):925-933.

26 Barabino S, Rolando M, Nardi M, Bonini S, Aragona P, Traverso CE. The effect of an artificial tear combining hyaluronic acid and tamarind seeds polysaccharide in patients with moderate dry eye syndrome: a new treatment for dry eye. Eur J Ophthalmol 2014;24(2):173-178.

27 Lee SY, Tong L. Lipid-containing lubricants for dry eye: a systematic review. Optom Vis Sci 2012;89(11):1654-1661.

28 Vicario-de-la-Torre M, Benítez-del-Castillo JM, Vico E, Guzmán M, de-Las-Heras B, Herrero-Vanrell R, Molina-Martínez IT. Design and characterization of an ocular topical liposomal preparation to replenish the lipids of the tear film. Invest Ophthalmol Vis Sci 2014;55(12):7839-7847.

29 Acar D, Molina-Martínez IT, Gómez-Ballesteros M, Guzmán-Navarro M, Benítez-Del-Castillo JM, Herrero-Vanrell R. Novel liposomebased and in situ gelling artificial tear formulation for dry eye disease treatment. Cont Lens Anterior Eye 2018;41(1):93-96. 